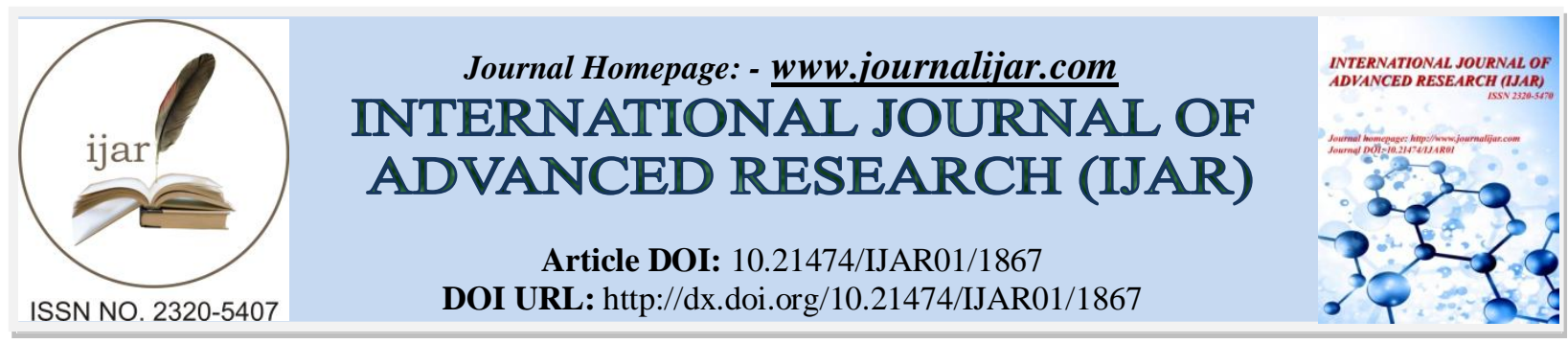

RESEARCH ARTICLE

\title{
NOVEL APPROACHES OF CONDIMENT EXTRACT SYNTHESIS OF NICKEL NANOPARTICLES FOR ANTIMICROBIAL ACTIVITIES: A GREEN EXPERTISE.
}

\author{
G.Pavan $^{1}$, M. Nagarajugoud ${ }^{2}$, S. Kalyani , P. Shireesha, M. Madhuri , N. Srikar and M. Abhilash \\ 1. University College of Science, Saifabad, Osmania University - 04, India. \\ 2. Govt. city college hyderabgad, high court road, near madina, Hyderabad 500002.
}

\section{Manuscript Info}

Manuscript History

Received: 12 August 2016

Final Accepted: 22 September 2016

Published: October 2016

Key words:-

Nanoparticle, SEM and TEM.

\section{Abstract}

The recent development and implementation of new technologies has led to new era of nano revolution which unfolds the role of plants in bio and green synthesis of nano materials [1].Metallic nanoparticles are being utilized in every phase of science along including medical fields and are still charming the scientists to explore new dimensions, generally attributed to their corresponding small sizes. The upcoming researches have proven their antimicrobial significance [2]. Although nanoparticles can be synthesized by an array of conventional methods, green route of synthesis is competent over physical and chemical methods [3].

In the present work nickel nanoparticles was synthesized using clove and extracts. The absorption maxima nickel is at $680 \mathrm{~nm}$. The morphology of the particles was confirmed by transmission microscopy (TEM) and Scanning electron microscopy (SEM). TEM results suggest the size of the particle is in the range of $50-200 \mathrm{~nm}$. SEM images show the particles are spherical and granular nature. The synthesized nanoparticles s how fairly good anti microbial activity.

Copy Right, IJAR, 2016,. All rights reserved.

\section{Introduction:-}

Nanobiotechnology is an enabling technology that deals with nanometer-sized materials in diverse fields of science such as biotechnology, nanotechnology, physics, chemistry and materials science. In addition to many physical and chemical methods which have been developed for preparing metallic nanoparticles, nanobiotechnology also serves as a significant technique in the progress of clean, non-toxic and environment friendly procedures for synthesis and assembly of metallic nanoparticles. The biosynthesis of nanoparticles has attracted attention of many researchers owing to their physical and chemical processes being expensive and drastic reaction consitions.

Nickel nanoparticles, due to their excellent physical and chemical properties and low cost of preparation have been of great interest. Copper nanoparticles havewide applications as heat transfer systems,antimicrobial materials, super strong materials, sensors and catalysts Nickel nanoparticles are very reactive because of with other particles [1] and increase their antimicrobial efficiency. Colloidal Nickel has been used as an antimicrobial agent for decades. Nickel nanoparticles $(2-5 \mathrm{~nm})$ have revealed a strong antibacterial activity and were able to decrease the microorganism concentration by $99.9 \%$. Due to the stability of Nickel nanoparticles supported on a matrix and their disinfecting properties. 


\section{Plant Description:-}

Cloves (Syzygium aromaticum) are the aromatic dried flower buds of a tree in the family Myrtaceae, The clove tree is an evergreen tree that grows to a height ranging from 8-12 $\mathrm{m}$, having large and sanguine flowers in numerous terminal clusters. The flowers buds are at first of pale colour and gradually become green after which they develop into a bright red, when they are ready for collecting. Cloves are harvested when $1.5-2 \mathrm{~cm}$ long and consist of a long calyx, terminating in four spreading sepals and four unopened petals which form a small ball in the center. Cloves (shown in Figure 1) are used in Indian Ayurvedic medicine, Chinese medicine and western herbalism and dentistry where the essential oil is used as an anodyne (painkiller) for dental emergencies. Cloves are used as a carminative, to increase hydrochloric acid in thestomach and to improve peristalsis. Cloves are also said to be a natural anthelmintic. The essential oil is used in aromatherapy when stimulation and warming are needed especially for digestive problems. Topical application over the stomach or abdomen are said to warm the digestive tract. Clove oil, applied to a cavity in a decayed tooth, also relieves toothache. Cloves may be used internally as a tea and topically as an oil for hypotonic muscles, including for multiple sclerosis. Some recommend avoiding more than occasional use of cloves internally in the presence of pitta inflammation such as is found in acute flares of autoimmune diseases. Eugenol (shown in Figure 2) comprises $72-90 \%$ of the essential oil extracted from cloves and is the compound most responsible for the cloves' aroma.

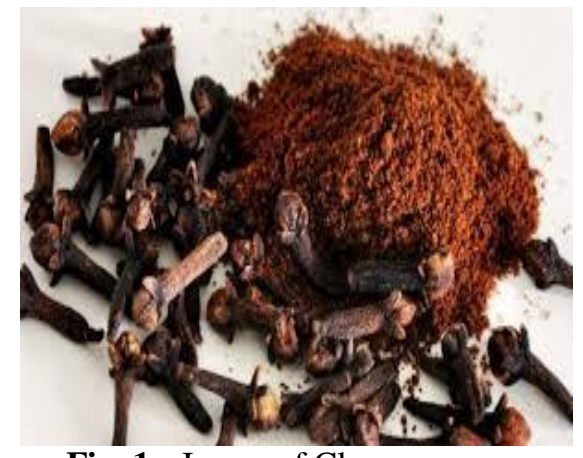

Fig. 1:- Image of Cloves.

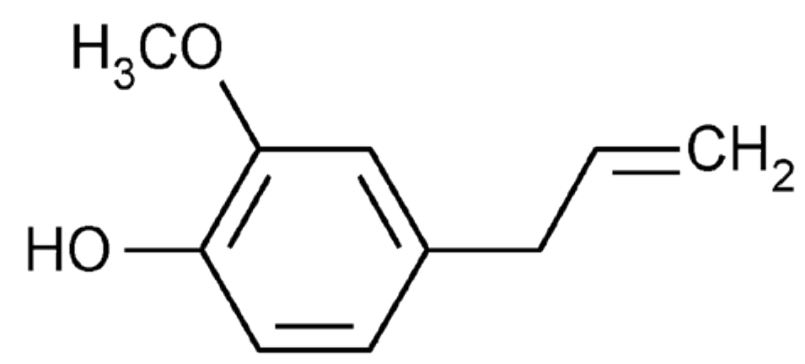

Fig. 2:- Chemical Structure of Compound Eugenol

\section{Materials and methods:- \\ Collection of Extracts:-}

Cloves were collected from the local market. They were washed and cleaned with triple distilled water and dried with water absorbent paper. Then it was crushed with the help of mortar and pestle dispensed in $10 \mathrm{ml}$ of sterile distilled water and heated for $2-3$ minutes at $70-80^{\circ} \mathrm{C}$. The extract was then filtered using Whatman's No.1 filter paper. The filtrate was collected in a clean and dried conical flask by standard sterilized filtration method and was stored.

\section{Synthesis of Nickel Nanoparticles:-}

For the synthesis of Nickel Nanoparticles, both the precursor and the reducing agent were mixed in a clean test tube in 1:1 proportion. For the reduction of $\mathrm{Ni}$ ions, $5 \mathrm{ml}$ of filtered Copper extract was mixed to $5 \mathrm{ml}$ of freshly prepared $0.001 \mathrm{M}$ aqueous of $\mathrm{Ni}\left(\mathrm{NO}_{3}\right)_{2}$ soiution it awas then kept for incubation for $1 \mathrm{hr}$. Within a particular time, the change in colour was noted from Dark brown to sea green Thus colour change indicates reduction and reduced Nickel nanoparticles were obtained. 


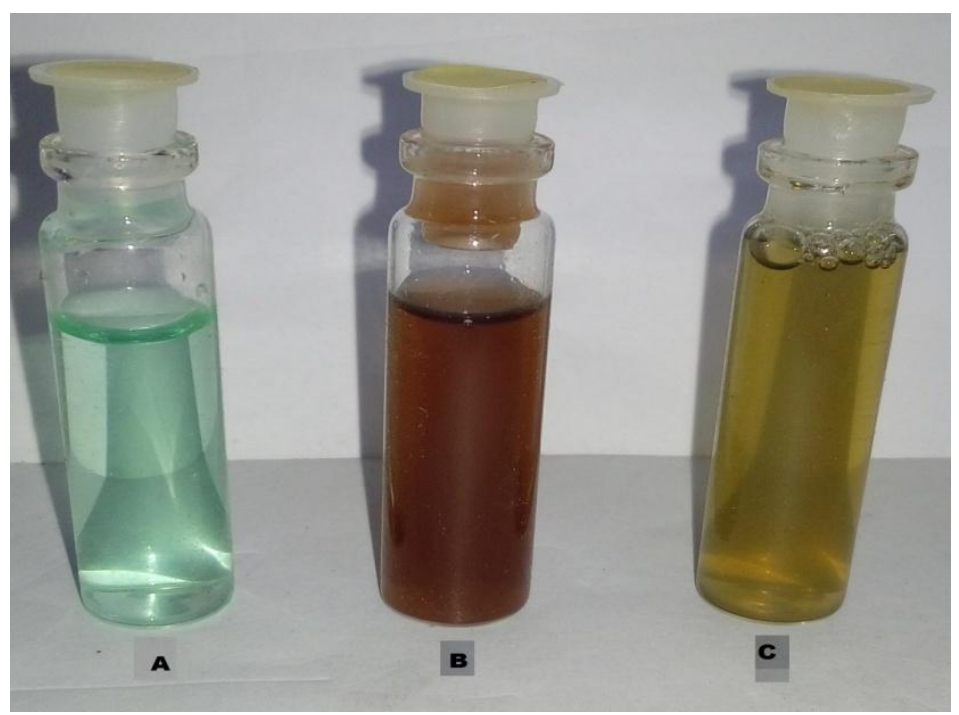

Fig.3:-

Tube A- contain nickel nitrate

Tube B- contain clove extract,

Tube C- contain sea greencoloured nickel nanoparticles solute

\section{pH Analysis:-}

The $\mathrm{pH}$ was determined by using Digital $\mathrm{pH}$ meter. The $\mathrm{pH}$ of the reduced solution with Nanoparticle meter. The $\mathrm{pH}$ of the reduced solution with Nanoparticle synthesized was found to be 1.89 .

\section{UV-Vis Spectra Analysis:-}

The reduction of pure $\mathrm{Ni}$ to nanoparticle was monitored by measuring the UV-Vis spectrum the most confirmatory tool for the detection of surface Plasmon resonance property (SPR) of NiNPs, by diluting a small aliquot of the sample in distilled water.UV-Vis spectral analysis was done by using UV-Vis UVVis spectral analysis was done by using is spectrophotometer Systronics 118 at the range of $650-680 \mathrm{~nm}$.

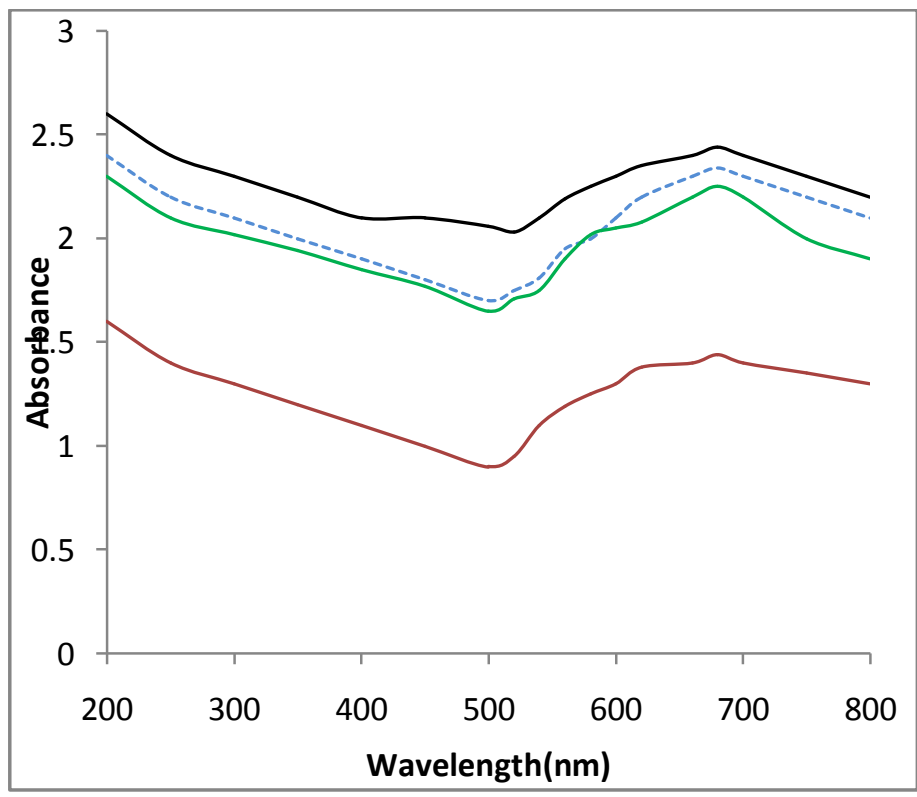

Fig.4:- UV-Vis Spectra for nickel nano particles. 


\section{Scanning Electron Microscopy:-}

The morphology of the as-prepared Ni productswas examined by FESEM. The typical SEM image shows that the product mainly consists of particle-like Ni nanoclusters with panoramicview and the size ranges from 150 to $200 \mathrm{~nm}$. However,further observation with high magnification reveals thatthese Ni nanoclusters are assembled by smallernanoparticles, which exhibit good uniformity and theaverage diameter is about $40 \mathrm{~nm}$. The average size Ofthese nanoparticles is about $40-45 \mathrm{~nm}$, almost in accordance with that from SEM observations.

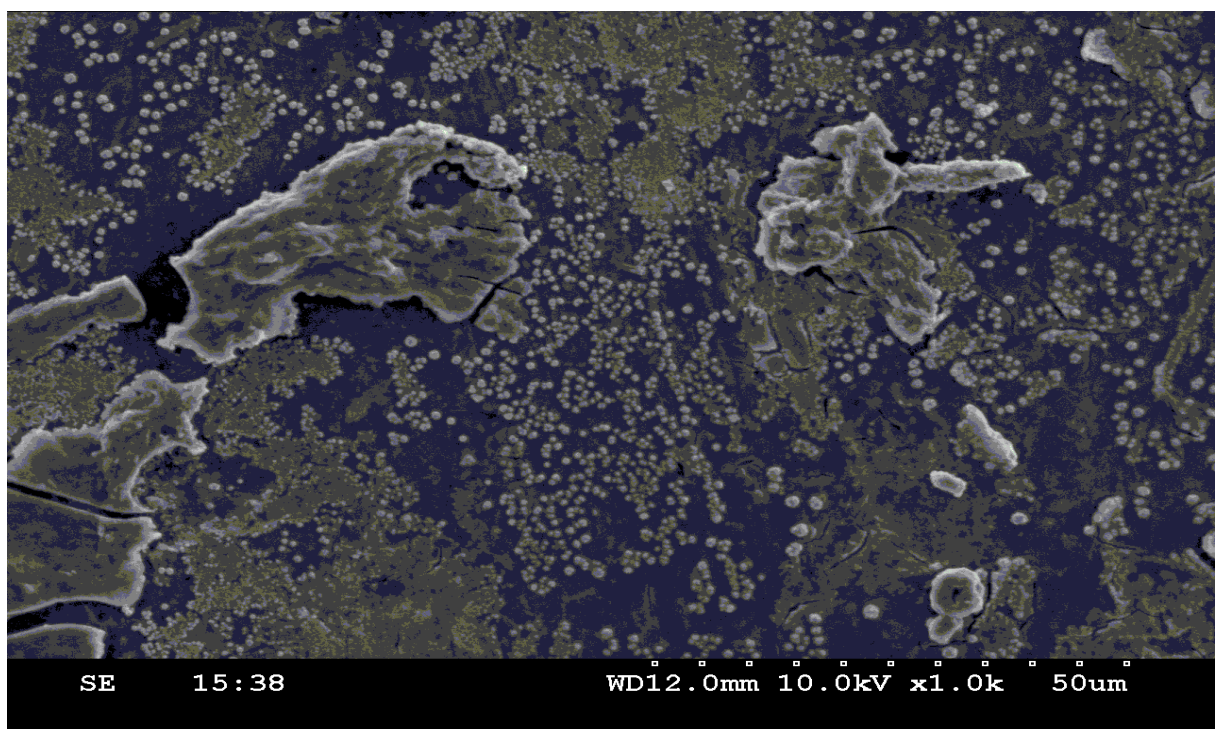

Fig.5:- SEM for nickel nano particles.

\section{Transmission Electron Microscopy:-}

The shape and size distribution of colloidal particles were characterized by transmission electron microscopy (TEM) two days after preparation. With a size range between 14 and $50 \mathrm{~nm}$ we can say that those particles are large and widely dispersed

\section{AntiMicrobial Activity:-}

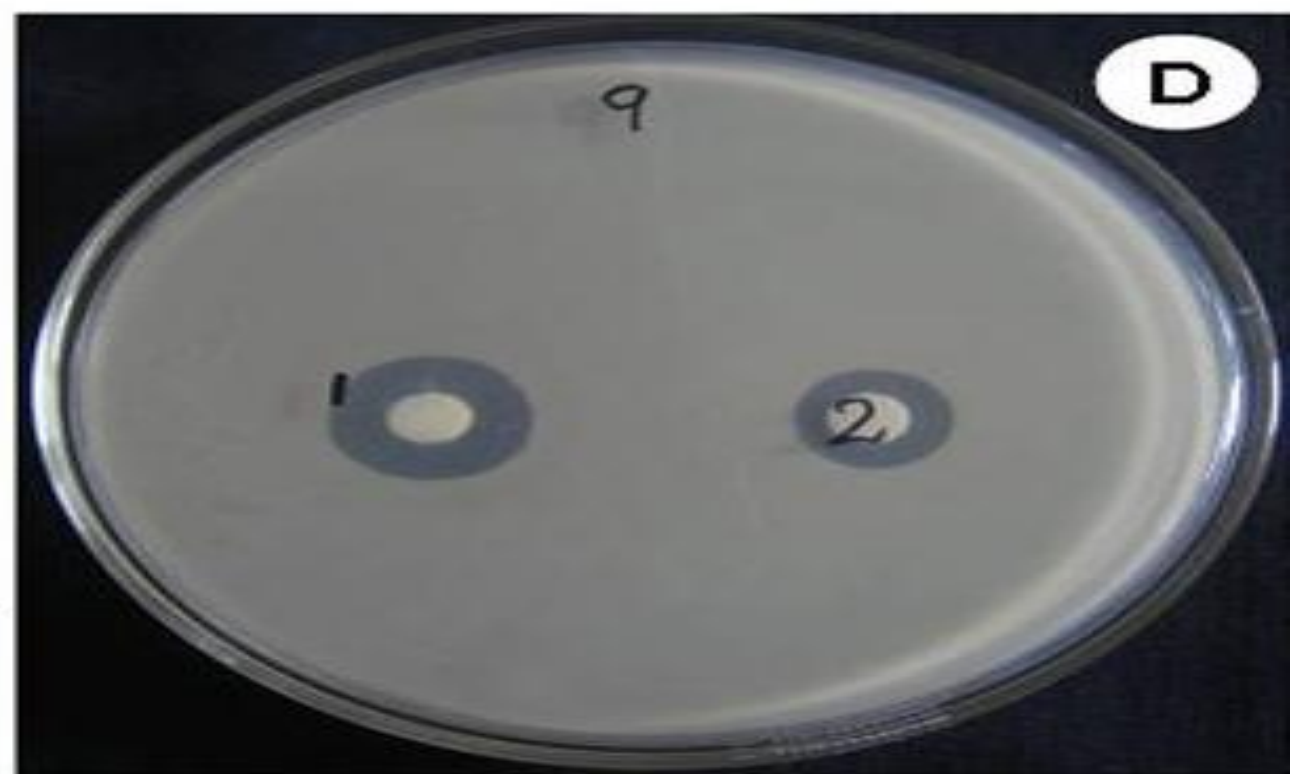

Nickel nano particles Showing antimicrobial activutry.e-coil Showing the zones of inbition 12nmand 9nm respectively . 


\section{Result and Discussion:-}

We have prepared spherical Ni nanoparticles inanoregime by a novel biological synthesis technique which is simple and environmentally benign. It is an easy,fast and cost effective technique and doesn't involve anyharmful and environmentally toxic chemicals used previously in conventional chemical reduction methods. Aqueous solutions of Ni nanoparticles with very good stability have been synthesized. It has been alsodiscussed that the biomolecules present in the biomass not only reduce the metal ions and but also stabilize themetal nanoparticles by preventing them from being oxidized after the preparation.

\section{Conclusion:-}

Biogenic synthesis of nickel nano particles using clove were performed by Brown to light green color. UV absorption studies of Ni nano particles showed the absorbance peak are $680 \mathrm{~nm}(\mathrm{Ni})$. The morphology of studies of particles was confirmed by transmission microscopy ( TEM ) and scanning electron microscopy ( SEM ). TEM results suggest the size of the particles is in the range of 50-200 nm.TEM images show the particles are spherical and granular nature. The synthesized nano particles show fairly good anti microbial activity.

\section{References:-}

1. "Green" Nanotechnologies: Synthesis of Metal Nanoparticles Using Plants.

2. V. V. Makarov, A. J. Love, O. V. Sinitsyna et al., Actanaturae (2014). 6 (1): 36 - 44.

3. Shakeel Ahmed, Mudasir Ahmad, BabuLal Swami, Saiqalkram. J. Adv. Res. (2016) 7: 1728.

4. Anamika Mubayi1, Sanjukta Chatterji1, Prashant M. Rai1,2, Geeta Watal1,*

5. Adv. Mat. Lett. 2012, 3(6), 519-525.

6. Athanassiou, E.K., Grass, R. N., \& Stark, W. J. (2007). Large scale production of carbon coated copper nanoparticles for sensor applications. Nanotechnology, 17, 1668-1673.

7. J. H. Fendler, Chem. Rev., 1987, 87, 877

8. G. Schmid, Chem. Rev., 1992, 92, 1709. 